This item was submitted to Loughborough's Research Repository by the author.

Items in Figshare are protected by copyright, with all rights reserved, unless otherwise indicated.

\title{
Why do aqueous surfactant solutions spread over hydrophobic substrates?
}

\section{PLEASE CITE THE PUBLISHED VERSION}

http://dx.doi.org/10.1016/j.cis.2010.04.001

\section{PUBLISHER}

(c) Elsevier

VERSION

AM (Accepted Manuscript)

\section{LICENCE}

CC BY-NC-ND 4.0

\section{REPOSITORY RECORD}

Starov, Victor, Natalia A. Ivanova, and Ramon Rubio. 2011. "Why Do Aqueous Surfactant Solutions Spread over Hydrophobic Substrates?”. figshare. https://hdl.handle.net/2134/7673. 
This item was submitted to Loughborough's Institutional Repository (https://dspace.lboro.ac.uk/) by the author and is made available under the following Creative Commons Licence conditions.

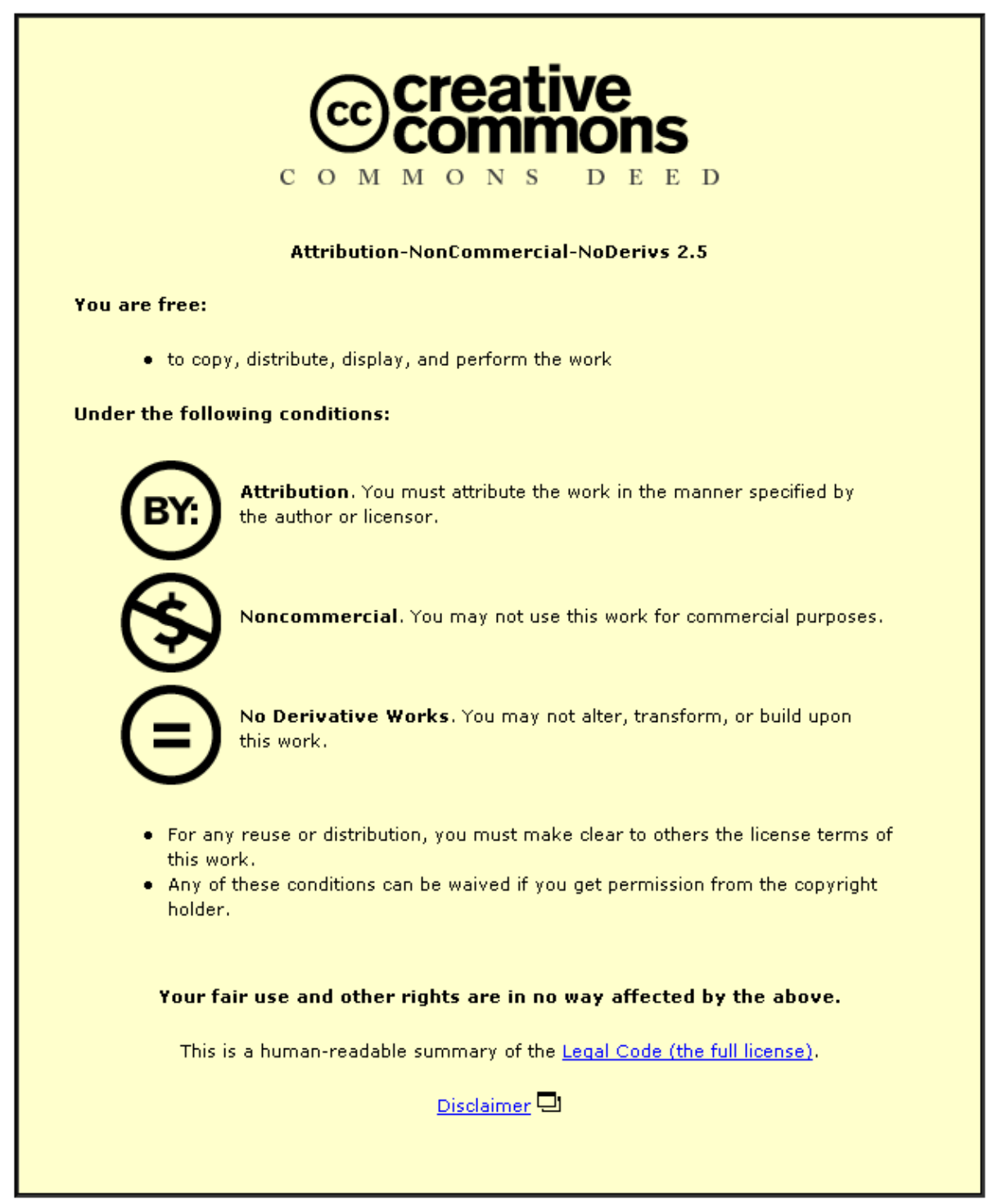

For the full text of this licence, please go to: http://creativecommons.org/licenses/by-nc-nd/2.5/ 


\title{
Why does aqueous surfactant solutions spread over hydrophobic substrates?
}

\author{
Victor Starov ${ }^{1 *}$, Natalia Ivanova ${ }^{1}$, Ramon Rubio ${ }^{2}$ \\ ${ }^{1}$ Department of Chemical Engineering, Loughborough University, \\ Loughborough, LE11 3TU, UK \\ ${ }^{2}$ Department of Quimica Fisica I, Faculty Quimica, Universidad Complutense, \\ 28040-Madrid, Spain
}

\begin{abstract}
Kinetics of spreading of aqueous surfactant solution droplets over highly hydrophobic substrates proceeds in one slow stage at concentration of surfactants below some critical value and in two stages if the surfactant concentration above the critical value: the fast and relatively short first stage is followed by a slower second stage. It is shown that the kinetics of spreading at concentrations below critical and the second stage at concentrations above critical are determined by a slow surfactant molecules transfer on a bare hydrophobic substrate in front of the moving three phase contact line (autophilic phenomenon). The latter process results in an increase of the interfgacial tension of the hydrophobic solid surface in front and the spreading as a result. It is proven that in spite of that the latter process results in a decrease of the total free energy of the droplet and, hence, the adsorption of surfactants molecules on a bare hydrophobic substrate in front of the moving three phase contact line is a spontaneous process. The duration of the first stage of spreading in the case of the surfactant concentration above the critical correlates well with the duration of adsorption of surfactant molecules onto liquid-air interface. The latter allows assuming that the adsorption on the liquid-air interface is the driving mechanism of spreading during the first fast stage of spreading at surfactant concentrations above the critical. It is discussed why the first stage does not present
\end{abstract}


in the case of surfactant concentrations below the critical concentration in spite of the longest duration of adsorption in this case.

*Corresponding author: V.M.Starov@Iboro.ac.uk

\section{Introduction}

Surfactant enhanced spreading of water over low energy surfaces is very important process in many industrial, pharmaceutical, agriculture and life science applications. The spreading dynamic of surfactant solutions has been extensively investigated over last years ${ }^{1-7}$; however an understanding of the mechanism of influence of surfactants on spreading of droplets of aqueous surfactant solutions is to be understood.

Under isothermal conditions and in the absence of external forces a liquid moves under the action of one of two the following forces: a pressure gradient and/or a surface tension gradient. In the case of pure liquids on smooth hydrophilic substrates the spreading behaviour obeys to the classical Tanner's law indicating that the combined action of capillary and Derjaguin's pressure (disjoining pressure), acting in a vicinity of the three phase contact line, is a driving force ${ }^{8}$. However, a mechanism behind the spreading of surfactant solutions is more complicated and has not been understood yet. No doubt that the surface forces (Derjaguin's or disjoining pressure) are involved but the presence of surfactants modifies those forces, causes the surface tension gradients and changes dramatically events in a vicinity of the three phase contact line. All those events in a vicinity of the three phase contact line are to be understood. It is the reason why below we try to understand the phenomenon of spreading of surfactants solutions over hydrophobic substrates using a simplified picture. 
The analysis of data published in the literature shows that spreading of surfactant solutions over hydrophobic surfaces proceeds at least in two stages with different spreading rates depending on hydrophobicity of substrates and concentration of surfactants. ${ }^{9-16}$ Obviously the spreading of surfactant solutions is affected by adsorption of surfactant molecules at interfaces, which could drastically changes interfacial tensions and energy balance at a three-phase contact line, promote the spreading of aqueous surfactant solutions: the rate of adsorption of surfactant molecules and depletion of surfactant at the expanding interface during the spreading reflects on the changing of spreading rate of droplet. ${ }^{17}$

The mechanisms proposed by a number of researchers ${ }^{4-7,10}$ for spreading of surfactant solution over hydrophobic surfaces relayed only on the adsorption processes on (i) the liquid-vapor and/or (ii) the solid-liquid interfaces, and completely excluded the possibility of adsorption of surfactant on (iii) the solid-vapour interface in front of the moving three phase contact line. The latter phenomenon has been suggested as a driving force of spreading of aqueous surfactant solutions over hydrophobic substrates ${ }^{18-24}$. Below we consider the latter mechanism and show that spreading of surfactant solutions on hydrophobic substrates involves a spontaneous adsorption of surfactant molecules in front of the moving three phase contact line and the latter phenomenon controls the rate of spreading of surfactant solutions over hydrophobic substrates.

\section{Experimental evidences and existing theoretical models}

The idea of the adsorption of surfactant molecules in front of moving three phase contact line on a bare hydrophobic substrate was put forward by Churaev and co-workers ${ }^{18-20}$ in their experiments on a spontaneous imbibition/rising of aqueous solutions of non-ionic surfactant in hydrophobized quartz capillaries. They 
found that a characteristic time scale of the imbibition process was several minutes and even hours, that is, presumably all adsorption processes on the liquid-air and the solid-liquid interfaces in capillaries of around $10 \mu \mathrm{m}$ in the diameter were likely to be over. The authors assumed that adsorption of surfactant molecules onto a bare hydrophobic surface in front of the moving three-phase contact line was the only possible explanation ${ }^{18-20}$. The latter process results in a partial hydrophilization of the hydrophobic substrate ahead of the moving liquid front of the aqueous surfactant solution and the latter leads to spontaneous penetration of the solution into the hydrophobic capillary (autophilic phenomenon). Later, Starov ${ }^{21}$ developed a theoretical model for the slow capillary rise of surfactant solutions in hydrophobic capillaries, which is based on the adsorption of surfactant molecules onto bare hydrophobic surface of capillary. Note that $i^{21}$ the time scale of the capillary rise was several hours.

The important feature of those processes of the spontaneous imbibitions/rise of aqueous surfactant solutions into hydrophobic capillaries is the time scale of the process $^{18-21}$ : adsorption of surfactant solutions in front of the moving three phase contact line was at equilibrium (in the case of the capillary imbibitions) or quasiequilibrium (in the case of the capillary rise) with the bulk surfactant concentration behind the moving meniscus.

For the first time the adsorption of surfactant molecules on a bare hydrophobic substrate in front of the moving three phase contact line was used to explain the kinetics of spreading of aqueous surfactant solutions over hydrophobic substrates in ref. ${ }^{22}$. Note, in this case the situation is substantially different from the case of imbibitions/rise of surfactant solutions into hydrophobic capillaries: the concentration of surfactants in front of the moving three phase contact line is not at 
the equilibrium with the bulk concentration of surfactants inside the droplet. If that concentration had been at the equilibrium with the bulk concentration in the droplet then the spreading process would have stopped. The latter means that the concentration of surfactants in front of the moving three phase contact line is not at the equilibrium and just relaxation to the equilibrium determines the rate and the duration of the spreading process. The latter have been confirmed later in ref. ${ }^{23}$.

In ref $^{25}$ a distribution of surfactant molecules in a vicinity of the three phase contact line was investigated using autoradiography method. It was shown that the surfactant molecules accumulates in some cases in a vicinity of the three phase contact line. However, the method used $\mathrm{in}^{25}$ did not allow separating the influence of evaporation (which proceeds mostly in a vicinity of the three phase contact line) and adsorption (autophilic phenomenon) in the same region.

The decisive experimental evidence of presence of surfactant molecules in front of a contact line on a hydrophobic surface has been provided by Kumar et $\mathrm{al}^{24}$. Their carefully designed experiments have proven the presence of more wettable areas ahead a moving three phase contact line as compared with a clean hydrophobic surface in the case of both advancing and receding three phase contact line. It has been shown ${ }^{24}$ that the local solid-vapor interfacial tension increases with an increasing bulk surfactant concentration. The latter results in the contact angle to be lower than expected from the reduction based on the solid-liquid and liquid-vapor surface tensions alone. Authors ${ }^{24}$ have referred to this phenomenon as the autophilic effect, which is used below.

A theoretical model of an evolution of contact angle and a diameter of the droplet base, which spreads over a hydrophobic surface has been proposed by Starov et al. ${ }^{23}$ According to this model the adsorption of surfactants in front of moving 
edge of a droplet is a driving force for spreading of droplet of aqueous surfactant solution. As far as the driving force is proportional to the difference $\Gamma(t)-\Gamma_{e}$, the adsorption behaviour is suggested to be described by the following kinetic equation:

$$
\frac{d \Gamma_{s v}(t)}{d t}=\alpha\left[\Gamma_{e, s v}-\Gamma_{s v}(t)\right],
$$

where $\Gamma_{s v}(t)$ is the instantaneous value of surfactant adsorption onto the solid surface in front of the droplet on the three-phase contact line, $\Gamma_{e, s v}$ is the equilibrium surface concentration of adsorbed surfactant molecules, and $\tau=\alpha^{-1}$ is the time scale of surfactant transfer from the droplet onto the solid-vapour interface in front of the moving three-phase contact line. The parameter $\alpha$ was expressed as follows ${ }^{23}$ :

$$
\alpha=\alpha_{T} \Gamma_{l v} \exp (-\Delta E / k T)
$$

where the $\alpha_{T}$ is determined by the thermal fluctuations only; $\Delta E$ is an energy barrier for surfactant transfer from the liquid drop onto the solid-liquid interface; $k$ and $T$ are Boltzman's constant and absolute temperature, respectively; $\Gamma_{l v}$ is a surfactant concentration on the liquid-air interface in a vicinity of the three phase contact line. The latter concentration is an increasing function of the bulk surfactant concentration inside the droplet with a maximum close to the critical micelle/aggregation concentration (CMC/CAC). Hence, according to Eq. (2) $\tau$ decreases with increasing of the bulk concentration of surfactant, while above the CMC $\tau$ should level off and reach its lowest value.

These theoretical assumptions have been supported by experiments for a case of a spreading of SDS surfactant solutions over hydrophobic substrates characterized by a contact angle $105^{\circ}$ of pure water. ${ }^{23}$ Later, Dutschk et al ${ }^{13,14}$ identified the autophilic mechanism as the only one possible explanation for a long- 
time regime of spreading of ionic and nonionic surfactant solutions over different polymeric substrates.

Estimation of possible values of the energy barrier in Eq. 920 is given below.

It was shown earlier ${ }^{15}$ that at low concentrations below CAC the kinetics of spreading is described by the theory presented $\mathrm{in}^{23}$. As we already mentioned above according to that theory the surfactant molecules adsorb in front of the moving three phase contact line. The latter process results in a partial hydrophilisation of an initially hydrophobic substrates and a spreading as a consequence. However, at higher concentrations both in between CAC and critical wetting concentration (CWC) and above CWC the spreading of T6 and T8 proceeds in two stages: the first faster stage that rate is more than ten time faster than the rate of the next much slower second stage. It is shown that the second stage develops according to the previously described model.

According to ${ }^{15,23}$ the dynamic of contact angle of a spreading droplet of surfactant solution over hydrophobic substrate at concentrations below CAC can be described as

$$
\ln y=-\alpha t, \quad y=\frac{\cos \theta_{\infty}-\cos \theta(t)}{\cos \theta_{\infty}-\cos \theta_{0}}
$$

that is the linear dependency, which can be easily experimentally verified. Figure 1 presents the comparison with the experimental data on spreading of T6 solution ${ }^{15}$.

It was found that the behaviour of $\cos \theta(t)$ was substantially different at concentrations below $\mathrm{CAC} / \mathrm{CMC}$ and above $\mathrm{CAC} / \mathrm{CMC}^{15}$. At concentrations below CAC/CMC behaviour of the $\cos \theta(t)$ in the case of Tween 20, T6 and T8 was in agreement with Eq. (3) (see Figure 1). However, in the case of concentrations above $\mathrm{CAC/CMC}$ and above CWC behaviour of $\cos \theta(t)$ was substantially different. At those 
higher concentrations two stages of spreading were detected: the first fast stage of spreading, which was followed by the second slower stage of spreading. Both stages were fitted using linear dependencies similar to Eq. (3). In this case those dependences were rewritten as

$$
\begin{aligned}
& \ln y=-\alpha_{1} t, \quad y=\frac{\cos \theta\left(t_{1}\right)-\cos \theta(t)}{\cos \theta\left(t_{1}\right)-\cos \theta^{0}} ; \quad 0<t<t_{1} \\
& \ln y=-\alpha_{2} t, \quad y=\frac{\cos \theta_{\infty}-\cos \theta(t)}{\cos \theta_{\infty}-\cos \theta\left(t_{1}\right)} ; \quad t_{1}<t
\end{aligned}
$$

where $t_{1}$ is the duration of the first fast stage of spreading. Linear dependences according to Eq. (4) fit reasonably well the experimental data at concentrations above the CAC/CMC and above the CWC (see Figures 2 and 3). However, Figures 2, 3 clearly shows that during the first stage of spreading the experimental dependencies do not follow exactly the straight line. However, it was attempted to fit this part by a straight line ${ }^{15}$. The later was done only for an estimation of the order of magnitude of the characteristic time scale of the fast first stage of spreading. However, the mechanism of spreading during the first stage remains unknown. Below we try to understand the mechanism of spreading during the fast first stage.

To summarize experimental findings presented $\mathrm{in}^{15}$ : the slow stage of spreading of aqueous trisiloxane surfactant solutions over highly hydrophobic PTFE AF coated silicon wafers is described in the frame of the proposed theory ${ }^{23}$, and a good agreement between experimental and predicted data has been found.

Figure $4(a-b)$ presents the experimental dependencies of the characteristic time of surfactant molecules transfer on bare hydrophobic substrates in front of the moving three phase contact line at different degrees of hydrophobicity of substrates. Figure $4(a-b)$ shows that the time of surfactant transfer reached constant values at concentrations close to the CWC for trisiloxane surfactants ${ }^{15}$ and close to the CMC 
for conventional surfactants. ${ }^{14}$ Note, to draw experimental data in Figure 4 (a-b) we used the values of the characteristic time for the slow second stage of spreading in the case of concentrations of surfactant inside the drops above CMC/CAC.

Figure 5 shows the comparisons between the experimental data on the time evolution of the diameter of the droplet base and theoretical predictions according to Eqs. (4). Both figures clearly demonstrate a reasonable agreement between the theoretical predictions based on the above theory and experimental data published in Refs. ${ }^{13-15,23}$. However, Figure 5 shows that the agreement is much better during the second stage of spreading than the agreement during the first fast stage of spreading.

\section{Excess free energy of an aqueous surfactant droplet}

Let us consider a free energy of a droplet on a solid substrate as it shown in Figure 6. Free energy of the system, $\Phi$, can be written as

$$
\Phi=\gamma_{l v} S+P V+\pi a^{2}\left(\gamma_{s l}-\gamma_{s v}\right),
$$

where $\gamma_{l v}, \gamma_{s l}$ and $\gamma_{s v}$ are interfacial tensions of the liquid-vapour, the solid-liquid and the solid-air, respectively; $S$ is the area of the liquid-vapour interface, $P$ is the excess pressure, $V$ is the liquid volume, $\theta$ is the contact angle. It is well known that the requirement of the equilibrium results in an equation for the droplet profile. The only solution of that equation is a spherical segment (see ref $^{1}$ for details). Expressing $P, S, a$ and $V$ via radius $R$ and the contact angle $\theta$ the following expressions can be deduced:

$$
P=-2 \gamma_{l v} / R, S=2 \pi R^{2}(1-\cos \theta), a^{2}=R^{2}\left(1-\cos ^{2} \theta\right), V=\frac{1}{3} \pi R^{3}\left(2-3 \cos \theta+\cos ^{3} \theta\right)
$$

Substitution of all above expressions into Eq. (5) results in 


$$
\Phi=\frac{\pi \gamma_{l v} R^{2}}{3} f(\theta) ; \quad f(\cos \theta)=\left(2-3 \cos \theta+\cos ^{3} \theta\right)
$$

Taking into account the assumption of constancy of the drop volume Eq. (6) can be rewritten as follows,

$$
\Phi=\frac{\gamma_{l v} \pi^{1 / 3} V^{2 / 3}}{3^{1 / 3}} f^{1 / 3}(\cos \theta) .
$$

Transversality condition at the equilibrium (see ref. ${ }^{1}$ for example) results in the wellknown Young equation $\cos \theta=\frac{\gamma_{l v}-\gamma_{s l}}{\gamma_{l v}}$, which in a combination with Eq. (8) yields the following equation for the free energy

$$
\Phi=\gamma_{l v}\left(\frac{\pi V^{2}}{3}\right)^{1 / 3} f^{1 / 3}\left(\frac{\gamma_{s v}-\gamma_{s l}}{\gamma_{l v}}\right)
$$

Eq. (9) gives the dependency of the excess free energy of the droplet via three interfacial tensions $\gamma_{l v}, \gamma_{S l}$ and $\gamma_{S v}$.

Let us calculate derivatives of the excess free energy of the droplet with all three interfacial tensions $\gamma, \gamma_{S l}$ and $\gamma_{S V}$. Simple calculations show that

$$
\begin{aligned}
& \frac{\partial \Phi}{\partial \gamma_{s l}}=A \frac{\sin ^{2} \theta}{f^{2 / 3}(\cos \theta)}>0, \\
& \frac{\partial \Phi}{\partial \gamma_{l v}}=A \frac{2(1-\cos \theta)}{f^{2 / 3}(\cos \theta)}>0, \\
& \frac{\partial \Phi}{\partial \gamma_{s v}}=-A \frac{\sin ^{2} \theta}{f^{2 / 3}(\cos \theta)}<0,
\end{aligned}
$$

where $A=\left(\frac{\pi V^{2}}{3}\right)^{1 / 3}$. 
Eqs. (10) and (11) show that the excess free energy of the droplet decreases if solid-liquid interfacial tension decreases and liquid-vapour interfacial tension decreases. These two conclusions are well known. As a result two spontaneous processes take place in the presence of surfactants: adsorption of surfactants on the liquid-hydrophobic solid interface and adsorption on liquid -vapour interface.

Eq. (12) shows that the excess free energy of the droplet decreases if the solid-vapour interfacial tension increases. Note, the adsorption of surfactant molecules on a bare hydrophobic surface in front of the three phase contact line (autophilic phenomenon) results in a local increases of the solid-vapour interfacial tension. However, according to Eq. (12) the total excess free energy decreases because of that process. The latter means that the adsorption of surfactant molecules on a bare hydrophobic substrate in front of the three phase contact line is a spontaneous process. It is interesting to note, that the latter simple conclusion has never been noticed earlier.

\section{Possible mechanism of surfactant molecule transfer on a bare hydrophobic} substrate

Let us assume that a droplet of aqueous surfactant solution on a highly hydrophobic substrate is not in the actual contact with the substrate (Figure 7a), but connected to the solid substrate through surfactant molecules: hydrophilic heads in the aqueous phase, hydrophobic tails attached to the hydrophobic solid substrate. Under the action of combined capillary and surface forces (Derjaguin's or disjoining pressure) the liquid profile deviates from the planar interface in the centre. All surfactant molecules are not immobile but undergo thermal fluctuations. Hence, the surfactants in an immediate vicinity of the plane interface (circled in Figure 7a) can jump onto the hydrophobic substrate in the course of fluctuation (Figure 7b). Three 
processes occur simultaneously after such jump: (i) a surface diffusion of surfactant molecules along the hydrophobic substrate away from the droplet. As a result of this process some surfactant molecules will leave the apparent three phase contact zone. This results in a partial hydrophilisation of the bare hydrophobic substrate in front of the droplet; (ii) a Marangoni flow, caused by the surface tension gradient. As a result a part of the free surface (a b) (Fig. 7b) will be covered by surfactant molecules; (iii) as a result of attraction between the hydrophilic parts of the molecules and the free surface $\left(\begin{array}{ll}a & b\end{array}\right)$ the resulting gap will be healed. This process results in an extension of the droplet base that is, spreading.

Note that all presented above consideration based on pictures (Figures 7a and b) may remain valid in both cases of non-wetting (contact angle more than $90^{\circ}$, Figure $8 \mathrm{a}$ ) and partial wetting (contact angle is less than $90^{\circ}$, Figure $8 \mathrm{~b}$ ).

As the autophilic phenomenon results in an increase of the surface energy locally, then the adsorption goes via a potential barrier, which can be estimated as follows. Let us consider a change in a local free energy caused by a jump of a single surfactant molecule from a liquid-vapour interface on a bare hydrophobic surface (Figs. $7 \mathrm{a}$ and $7 \mathrm{~b})$. The associated energy barrier is:

$$
\Delta E=A_{s v}\left[\gamma_{s v}\left(\Gamma_{s v}\right)-\gamma_{s v}(0)\right]-A_{l v}\left[\gamma_{l v}\left(\Gamma_{l v}\right)-\gamma_{l v}(0)\right]
$$

where $A_{s v}$ and $A_{l v}$ are the surface areas of surfactant molecule at the solid-vapor and the liquid-vapor interfaces, correspondingly; $\gamma_{s v}(0)$ is the interfacial surface tension of the bare hydrophobic solid surface; $\gamma_{l v}(0)$ is the surface tension of pure water on the liquid-vapor interface; $\gamma_{s v}\left(\Gamma_{s v}\right)$ and $\gamma_{l v}\left(\Gamma_{l v}\right)$ are the interfacial tensions 
at the solid-vapor and the liquid-vapor interfaces with adsorbed surfactants molecules, correspondingly.

Let us estimate the energy barrier for aqueous solution of $\mathrm{C}_{12} \mathrm{E}_{8}$ at $\mathrm{CAC}$ on OTS surface using data presented by Kumar et al. ${ }^{24}$. Using the required data for $\mathrm{C}_{12} \mathrm{E}_{8}$ from refs. $^{17,24}$ we conclude: $\gamma_{s v}\left(\Gamma_{s v}\right)-\gamma_{s v}(0) \approx 24.5 \mathrm{mN} / \mathrm{m}$, $\gamma_{l v}\left(\Gamma_{l v}\right)=35.2 \mathrm{mN} / \mathrm{m}$ is the liquid-vapor surface tension, and $A_{l v}=62 \AA^{2}$ is the area per molecule. Assuming that $A_{s V}=A_{l v}{ }^{24}$ Eq. (13) gives us the following value of the local energy barrier: $\Delta E \approx 10 \mathrm{kT}$. The latter estimation shows that the energy barrier for the particular case considered is not unreasonably high. However, the height of the barrier determines the slow rate of the surfactant molecule transfer and the slow rate of spreading determined by that mechanism.

Kinetics of spreading during the first faster stage of spreading of surfactant solutions: quasi-steady state approach

It is well known that at low capillary numbers ${ }^{1} C a<1$, where $C a=\frac{\mu U}{\gamma_{l v}}, \mu$ is the dynamic viscosity, $U=d a / d t$ is the velocity of spreading, where $t$ is time, the drop shape remains the spherical form (Figure 6) and varies quasi-steadily with $a(t)$. We assume below that $a(t)<(\gamma / \rho g)^{1 / 2}$, where $\rho$ is the liquid density. That is the gravity action can be neglected.

Using the expression for volume and radius of the droplet base from Eq. (6) we can express the radius of the droplet base, $a(t)$, in the following form

$$
a(t)=\frac{V^{1 / 3}}{\varphi^{1 / 3}(\cos \theta(t))}, \quad \varphi(\cos \theta(t))=\frac{\pi}{3} \frac{2-3 \cos \theta(t)+\cos ^{3} \theta(t)}{\left(1-\cos ^{2} \theta(t)\right)^{3 / 2}}
$$


If the evaporation of liquid can be neglected, that is $V=$ const, differentiation with time of the latter equation results in

$$
\frac{d a}{d t}=\left(\frac{3 V}{\pi}\right)\left[\left(1-\cos ^{2} \theta\right)^{1 / 2}(1-\cos \theta)^{3 / 2}(2+\cos \theta)^{4 / 3}\right]^{-1} \frac{d \cos \theta}{d t} .
$$

This equation shows that all possible models of spreading of surfactant solutions can be reduced to the investigation of the behaviour of $\cos \theta(t)$, that is the contact angle on time.

The usually adopted assumption is as follows: Young equation is still valid in the course of spreading. The next step is an assumption that all three interfacial tensions in Young equation are completely determined by the adsorption at the corresponding interfaces

$$
\gamma_{l v}=\gamma\left(\Gamma_{l v}\right), \quad \gamma_{s v}=\gamma\left(\Gamma_{s v}\right), \quad \gamma_{s l}=\gamma\left(\Gamma_{s l}\right),
$$

where $\Gamma_{l v}, \quad \Gamma_{s v}, \quad \Gamma_{s l}$ are adsorption of surfactants on the liquid-vapour, the solidvapour, the solid-liquid interfaces, respectively. Taking into account the latter assumption Eq. (15) for the spreading can be rewritten as follows:

$$
\frac{d a}{d t}=B \frac{\left(\frac{d \gamma_{S V}}{d \Gamma_{s V}} \frac{d \Gamma_{S V}}{d t}-\frac{d \gamma_{s l}}{d \Gamma_{s l}} \frac{d \Gamma_{s l}}{d t}\right) \gamma_{l v}(\Gamma)-\left(\gamma_{s V}\left(\Gamma_{S V}\right)-\gamma_{s l}\left(\Gamma_{s l}\right)\right) \frac{d \gamma_{l v}}{d \Gamma} \frac{d \Gamma_{l v}}{d t}}{\gamma_{l v}^{2}(\Gamma)}
$$

where $B=\left(\frac{3 V}{\pi}\right)\left[\left(1-\cos ^{2} \theta\right)^{1 / 2}(1-\cos \theta)^{3 / 2}(2+\cos \theta)^{4 / 3}\right]^{-1}>0$. Note that all interfacial tensions and adsorptions in the latter equation are taken in a vicinity of the three phase contact line. In this case the derivative in the right hand site of the latter equation can be rewritten as

$$
\frac{d a}{d t}=B\left(I_{1}+I_{2}\right)
$$


where

$$
\begin{aligned}
& I_{1}=\frac{\left(\frac{d \gamma_{s v}}{d \Gamma_{s v}} \frac{d \Gamma_{s v}}{d t}-\frac{d \gamma_{s l}}{d \Gamma_{s l}} \frac{d \Gamma_{s l}}{d t}\right)}{\gamma_{l v}(\Gamma)} \\
& I_{2}=\frac{-\left[\gamma_{s v}\left(\Gamma_{s v}\right)-\gamma_{s l}\left(\Gamma_{s l}\right)\right] \frac{d \gamma_{l v}}{d \Gamma_{l v}} \frac{d \Gamma_{l v}}{d t}}{\gamma_{l v}{ }^{2}\left(\Gamma_{l v}\right)}
\end{aligned}
$$

We assume below that the bulk concentration of surfactants inside the droplet remains constant.

As we showed above $\frac{d \gamma_{s v}}{d \Gamma_{s v}}>0$, and it is well known that $\frac{d \gamma_{s l}}{d \Gamma_{s l}}<0$. Obviously both $\frac{d \Gamma_{S V}}{d t}>0$ and $\frac{d \Gamma_{S l}}{d t}>0$. Hence, $I_{1}>0$ is always positive. It is also obvious that $\frac{d \gamma_{l v}}{d \Gamma_{l v}}<0, \frac{d \Gamma_{l v}}{d t}>0$, hence $I_{2}>0$ at $\left[\gamma_{s v}\left(\Gamma_{S v}\right)-\gamma_{s l}\left(\Gamma_{S l}\right)\right]>0$ (or at $\left.\theta<90^{0}\right)$, that is adsorption on the liquid-vapour interface promote the further spreading, and $I_{2}<0$ at $\left[\gamma_{s V}\left(\Gamma_{s V}\right)-\gamma_{s l}\left(\Gamma_{s l}\right)\right]<0$ (or at $\left.\theta>90^{\circ}\right)$, that is, adsorption on the liquid-vapour interface does not favour the spreading if $\theta>90^{\circ}$. The conclusion following from the above analysis is rather surprising: the common believe is that the lower the liquidvapour interface the better wetting conditions. However, the latter is valid for the case when $\theta<90^{\circ}$ and not valid at $\theta>90^{\circ}$.

The latter observation explains the presence of the first faster stage in the case of concentrations above CAC (when $\theta<90^{\circ}$ ) and absence of the fast stage of spreading in the case of concentrations below $\operatorname{CAC}^{15}$ (when $\theta>90^{\circ}$ ). In the case of concentrations of trisiloxanes below CAC the initial and final contact angles observed in ${ }^{15}$ were above $90^{\circ}$. The latter means that the kinetics of adsorption of surfactant on 
the liquid-vapour interface and decreasing of liquid-vapour interface did not promote the spreading, but just the opposite tried to shrink the solid-liquid base. Note, the latter was impossible because of the hysteresis of contact angle. Note also, that the kinetics of adsorption in this case was the slowest.

However, in the case of concentrations above CAC the final contact angle was always below $90^{\circ}$. Hence, the adsorption on the liquid-vapour interface promote the spreading according to Eq. (18). The kinetics of adsorption on liquid-vapour interface was much faster than the corresponding adsorption on the solid-vapour interfaces. Hence, we can omit the term $\frac{\frac{d \gamma_{s v}}{d \Gamma_{s v}} \frac{d \Gamma_{s v}}{d t}}{\gamma_{v v}(\Gamma)}$ in Eq. (17). The kinetics of spreading is now described by the following equation:

$$
\frac{d a}{d t}=B \frac{\left(-\frac{d \gamma_{s l}}{d \Gamma_{s l}} \frac{d \Gamma_{s l}}{d t}\right) \gamma_{l v}(\Gamma)-\left(\gamma_{s v}\left(\Gamma_{s v}\right)-\gamma_{s l}\left(\Gamma_{s l}\right)\right) \frac{d \gamma}{d \Gamma} \frac{d \Gamma}{d t}}{\gamma_{l v}^{2}(\Gamma)},
$$

that is, determined by the kinetics of adsorption on the liquid-vapour and liquid-solid interfaces. We assume below that the latter two kinetics proceed on approximately equal time scales.

Now we compare the spreading dynamic of trisiloxane droplets with the corresponding dynamics of surface tension. The latter comparison allows us to clarify the relationship between the dynamics of surface tension at the liquid-air interface and experimental results on a contact angle evolution of droplet during the first fast stage of spreading found earlier ${ }^{15}$.

Figure 9 shows a dynamics of surface tension of trisiloxane T8 solutions in a wide range of concentrations starting from $0.2 \mathrm{CAC}$ to $20 \mathrm{CWC}$ from short to long 
adsorption times. The CWC (critical wetting concentration) ${ }^{11,15,27}$ is almost one order of magnitude higher than the CAC. Figure 9 shows that the whole adsorption process can be roughly subdivided into three stages: (i) initial stage, during that stage surface tension remains almost constant. Below we referee to the duration of that stage as "a lag time". The lag time decreases at the bulk concentration of surfactant increases and becomes undetectable at high concentrations: at concentrations above CAC the lag time was very small and could not be detected during measurements; (ii) the second dynamic stage. During that stage the surface tension decreases from its initial value to its lowest value. We referee below to the duration of that stage as "an adsorption time"; (iii) during the last third stage the adsorption remains constant.

In our spreading experiments small droplets of aqueous solutions of trisiloxane T8 spread over hydrophobic surfaces of PTFE AF coated silicone wafers. Full experimental procedure is described in details in Refs ${ }^{15,27-28}$.

Figure 12 shows contact angles dynamics of those solutions on PTFE AF surfaces. The latter figure shows that the whole spreading process can be roughly subdivided into three stages: (i) initial stage, during that stage the contact angle remains almost constant (a lag time). The lag time decreases at the bulk concentration increase and becomes undetectable at high concentrations: at concentrations close and above CWC the lag time was very small and could not be detected during measurements; (ii) the second dynamic stage. During that stage the contact angle decreases from its initial value to its lowest value during first stage; (iii) during the next third stage the contact angle changes according to the previous theory ${ }^{21}$, that is, surfactant molecules adsorb in front of the moving three phase contact line. 
A comparison between the dynamics of the surface tension (Figures 9) and the contact angle evolution for T8 (Figure 10) on PTFE AF surfaces reveals a similarity of their dynamic behaviour both qualitatively and quantitatively. Figure 10 shows that at low concentration $(C<C A C)$ spreading starts in around $2 \mathrm{~s}$ time lag after the droplet was deposited on the substrate, and takes around $100 \mathrm{~s}$ to reach an equilibrium contact angle. Figure 9 indicates that the surface tension decreasing starts after a shorter time lag as compared with the time lag at spreading; however, the time required for surface tension equilibration at those concentrations is comparable with the spreading time (see Figure 11). It was shown earlier ${ }^{15}$ that at concentrations below CAC the spreading proceeds in only one slow stage, which is in agreement with the consideration above (contact angle is above $90^{\circ}$ and adsorption on the liquid-air interface does not promote the spreading process). Hence, the spreading process is governed by adsorption of surfactant monomers in front of the moving three-phase contact line (TCL), which occurs from the liquidvapor interface in a vicinity of $\mathrm{TCL}^{23}$. Therefore, the time lag of spreading could be explained by a slow adsorption at the liquid-vapor interface that supplies molecules to be transported in front of TCP.

With increasing concentration $(\mathrm{C}>\mathrm{CAC} / \mathrm{CWC})$ the adsorption at the interface proceeds much faster (Figure 9): no time lag is detected and surface tension attains the equilibrium values in the time ranging $0.1-1 \mathrm{~s}$ depending on the bulk concentration.

In the spreading experiments at similar concentrations it was found ${ }^{15}$ that at such high concentrations spreading proceeds in two stages: a first short stage followed by a second long-time stage. Although the second stage of spreading was found to be well described by autophilic model, ${ }^{15,23}$ the physical nature behind the 
first fast stage remained unknown. However, comparison of the data presented in Figures 9 and 10 allows us to conclude that the duration of the first fast stage of spreading is very close to the duration of surface tension equilibration at concentration above CAC/CWC (see Figure 11). The latter probably means that the first fast stage of spreading is controlled by the adsorption of surfactant molecules on the liquid-vapour interface.

Note, however, that in ref [18-20] two stages of imbibitions were observed in the case of a capillary imbibitions of surfactant solutions into hydrophobic capillaries. The first faster stage of the imbibitions was attributed to the disintegration of micelles. We discussed above that the imbibitions process is substantially different from the spreading over hydrophobic substrates. However, in the case of spreading the faster stage of spreading has been detected at concentrations above CAC only [15]. That is, we cannot claim that the presence of aggregates does not contribute (or even determine) to the spreading process.

It is also important to notice that at concentrations above the CAC (see Figure 9) the values of surface tensions fall down to below $60 \mathrm{mN} / \mathrm{m}$ even at the moment of very beginning of measurement (due to the extremely fast adsorption) that is very clear reflected in the contact angle dynamics (Figure 10): with increasing concentration an initial contact angle of droplet decreases.

\section{Conclusions}

Kinetics of spreading of aqueous surfactant solution droplets over highly hydrophobic substrates proceeds in one slow stage at concentration of surfactants below CAC/CMC and in two stages if the surfactant concentration above the CAC/CMC: the fast and relatively short first stage is followed by a slower second stage. It is 
shown that the kinetics of spreading at concentrations below $\mathrm{CAC} / \mathrm{CMC}$ and the second stage at concentrations above $\mathrm{CAC/CMC}$ are determined by a slow surfactant molecules transfer on a bare hydrophobic substrate in front of the moving three phase contact line (autophilic phenomenon). The latter process results in an increase of the interfacial tension of the hydrophobic solid surface in front and the spreading as a result. It is proven that in spite of that increase of the local interfacial energy the latter process results in a decrease of the total free energy of the droplet. Hence, the adsorption of surfactants molecules on a bare hydrophobic substrate in front of the moving three phase contact line is a spontaneous process. The duration of the first stage of spreading in the case of the surfactant concentration above $\mathrm{CAC} / \mathrm{CMC}$ correlates well with the duration of adsorption of surfactant molecules onto liquid-air interface at the same concentrations. The latter allows assuming that the adsorption on the liquid-air interface is the driving mechanism of spreading during the first fast stage of spreading at surfactant concentrations above CAC/CMC. It is shown why the first stage does not take place in the case of surfactant concentrations below $\mathrm{CAC} / \mathrm{CMC}$ in spite of the longest duration of adsorption in this case.

\section{Acknowledgement}

This research was supported by Engineering and Physical Sciences Research Council, UK (Grant EP/D077869/1) and by EU under Grant MULTIFLOW, FP7-ITN- 2008-214919. The work of R.G. Rubio was supported in part by MEC through grant FIS2006-12281-002-01, and by CAM through project INTERFASES (S-0505/MAT-0227). 


\section{References}

1. Starov V., Velarde M., Radke C. "Dynamics of wetting and spreading". Taylor\&Frances, in "Surfactant Sciences Series", v.138, 2007.

2. Lee K.S., Ivanova N., Starov V.M., Hilal N., Dutschk V. Adv. Coll. Interface Sci., 2008. 144(1-2), 54-65

3. Joanny J.F. J. Coll. Interface Sci., 1989. 128, 407-415.

4. Ruckenstein E. J. Coll. Interface Sci., 1996. 179, 136-142.

5. Eriksson J., Tiberg F., Zhmud B. Langmuir, 2001. 17, 7274-7279.

6. Chengara A., Nikolov A., Wasan D. Coll. Surf A. 2002. 206, 31-39.

7. Chauhan A., Svitova T., Radke C. J. Colloid Interface Sci., 2000. 222, 221-232. 8.

8. V.M. Starov, V.V. Kalinin, J.-D. Chen. Advances Colloid \& Interface Sci., 50, 187$222(1994)$

9. Lee K.S., Starov V.M. J. Coll. Interface Sci., 2007. 314(2), 631-642;

10. von Bahr M., Tiberg F., Zhmud B. Langmuir 1999. 15(20), 7069-7075; von Bahr M., Tiberg F., Yaminsky V. Col. and Surf. A. 2001. 193, 85-96.

11. Svitova T., Hill R.M., Smirnova Yu., Stuermer A., Yakubov G. Langmuir, 1998, $14,5023-5031$.

12. Svitova T., Hill R., Radke C. Coll. Surf A. 2001. 183-185, 607-620.

13. Dutschk V, Sabbatovskiy K.G, Stolz M, Grundke K, Rudoy V.M. J. Colloid Interfaces Sci., 2003, 267, 456-.

14. Dutschk V. Breitzke B. Tenside Surf. Det. 2005, 42 (2), TS_2005_02_100238

15. Ivanova N., Starov V., Johnson D., Hilal N., Rubio R. Langmuir, 2009, 25 (6), 3564-3570.

16. Yang X., Dong J., Li X. J. Coll. Interface Sci. 2008, 325, 223-227.

17. Kumar N., Couzis A., Maldarelli C. J. Colloid Interfaces Sci, 2003, 267, 272-285. 
18. Berezkin V.V., Zorin Z.M., Frolova N.V., Churaev. N.V. Colloid Journal, 1975, 37, 1040-1044 (in Russian).

19. Berezkin V.V., Zorin Z.M., Iskandaryan G.A., Churaev. N.V. Colloid \& Polymer Sci., 1979, 267, 968-972 (in Russian).

20. Churaev, N.V., Martynov G.M., Starov V.M., Zorin Z.M. Colloid \& Polymer Sci, 1981, 259(7), 747-752.

21. Starov V. J. Coll. Interface Sci., 2004, 270, 180-186.

22. V.M. Starov, A.Yu. Maslov, G. Iskanderjan. A Slow Spreads of Surfactant Solutions over Hydrophobic Surfaces. Colloid. J. of Russian Academy of Sciences, 54(3) (1993), 410-418 [138-140].

23. Starov V., Kosvintsev S., Velarde M. J. Colloid and Interface Sci., 2000, 227(1), 185-190.

24. Kumar N., Varanasi K., Tilton R.D., Garoff S. Langmuir, 2003, 19 (13), 53665373.

25. O. Soboleva, V. Korobkov, B. Summ, V. Dolzhikova, G. Badun, A. Abramov. Colloid J., Russian Academy of Sciences, English Translation, 1998, 60 (6), 826-830 (Russian version).

26. Although both the solid-vapour and the liquid-vapour surface area of molecule are not the same, a difference between them is expected to be in the range of accuracy of measurements performed by different methods Refs. $11,17$.

(27) Ivanova N., Starov V., Rubio R., Ritacco H., Johnson D., Hilal N., Coll. Surf. A, 2009 (in press).

(28) Wei Ping C.C., Ivanova N., Starov V., Hilal N., Jpohnson D. Colloid J. (Russian Academy of Sciences, English Translation, 2009, 71 (3), 1. 


\section{Figure legends}

Fig. 1. Dependency of logarithm of $y=\frac{\cos \theta_{\infty}-\cos \theta(t)}{\cos \theta_{\infty}-\cos \theta_{0}}$ on spreading time according to Eq. (2) for T6 solution at concentration 0.2 CAC. Fitted straight line according to Eq. (3).

Fig. 2. Dependency of logarithm of $y$ on spreading time according to Eq. (4) for T6 solution at concentration 0.25 CWC. Fitted straight lines according to Eq. (4).

Fig.3. Dependency of logarithm of $y$ on spreading time according to Eq. (4) for T6 solution at concentration 5 CWC. Fitted straight lines according to Eq. (4).

Figure 4. Dependencies of the time of surfactant transfer on a bare hydrophobic substrate in front of the three phase contact line on bulk concentration of surfactants: (a) trisiloxane surfactants $\left(T_{4}, T_{6}\right.$ and $\left.T_{8}\right)$ on PTFE AF coated silicone wafers; (b) (redrawn from Ref. ${ }^{14}$ ) conventional ionic (SDS, DTAB) and non-ionic $\left(\mathrm{C}_{12} \mathrm{E}_{5}\right)$ surfactants on different polymers.

Figure 5. Time evolution of the droplet diameters for $T_{7.5}, T_{6}$, and $T_{4}$ solutions at concentrations close to the CWC (a second stage of spreading). Solid lines according to theoretical predictions (Refs. ${ }^{15,23}$ ) using the fitted values of the time of surfactant transfer.

Figure 6. Liquid droplet 1 on a solid substrate 2. a- radius of the droplet base, $\boldsymbol{R}$ radius of the curvature, $\boldsymbol{H}$-height, $\boldsymbol{\theta}$ - contact angle.

Figure 7a. A droplet of aqueous surfactant solution on a highly hydrophobic substrate. 1 - droplet, 2 - solid substrate. Hydrophobic tails of circled surfactant molecules attracted to the hydrophobic substrate and as results of thermal 
fluctuations some of those molecules can jump down on the bare hydrophobic substrate.

Figure $7 \mathrm{~b}$. Jump of a surfactant molecule from the liquid-air interface onto the solidair interface caused by thermal fluctuations. Three processes starts simultaneously but with different characteristic time scales: 1- surface diffusion along the solid surface, 2- Marangoni flow caused by the surface tension gradient, 3- attraction of the free part of the surface $(a b)$ and hydrophilic parts of the surfactant molecules underneath.

Figure 8a. Transition region from flat region in the centre to the droplet in the case of contact angle $\theta>90^{\circ}$.

Figure 8b. Transition region from flat region in the centre to the droplet in the case of contact angle $\theta<90^{\circ}$.

Figure 9. Dynamic surface tensions for T8 solutions at $298.15 \mathrm{~K}$ as a function of time. The symbols are experimental data, and the lines are eye-guides.

Figure 10. Dynamic contact angle for T8 solutions.

Figure 11. Dependency of the time of the surface tension equilibration for T8 $(\bullet)$, the duration of the fist stage of spreading droplets on PTFE AF coated silicon wafers from $\operatorname{ref}^{15}(\diamond)$. 
Time, sec

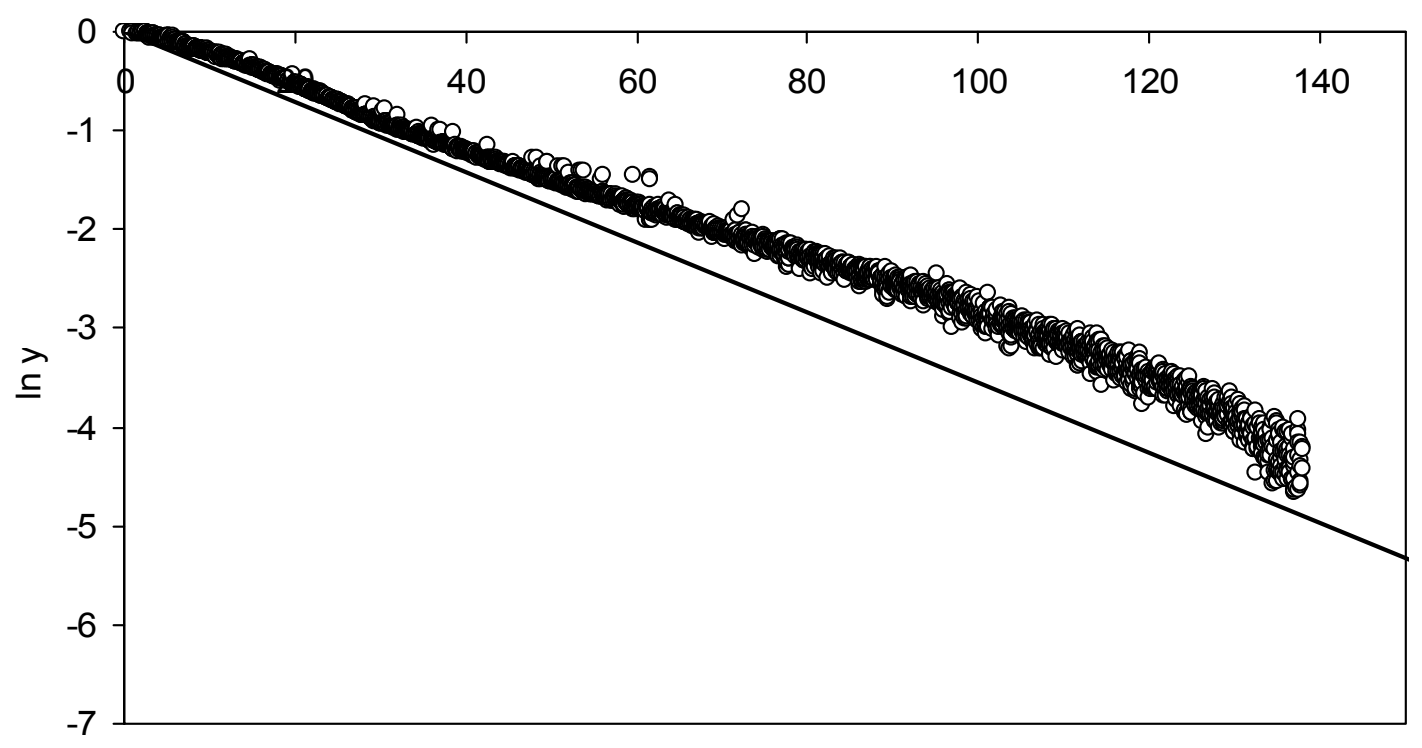

Fig. 1. Dependency of logarithm of $y=\frac{\cos \theta_{\infty}-\cos \theta(t)}{\cos \theta_{\infty}-\cos \theta_{0}}$ on spreading time according to Eq. (2) for T6 solution at concentration 0.2 CAC. Fitted straight line according to Eq. (3). 


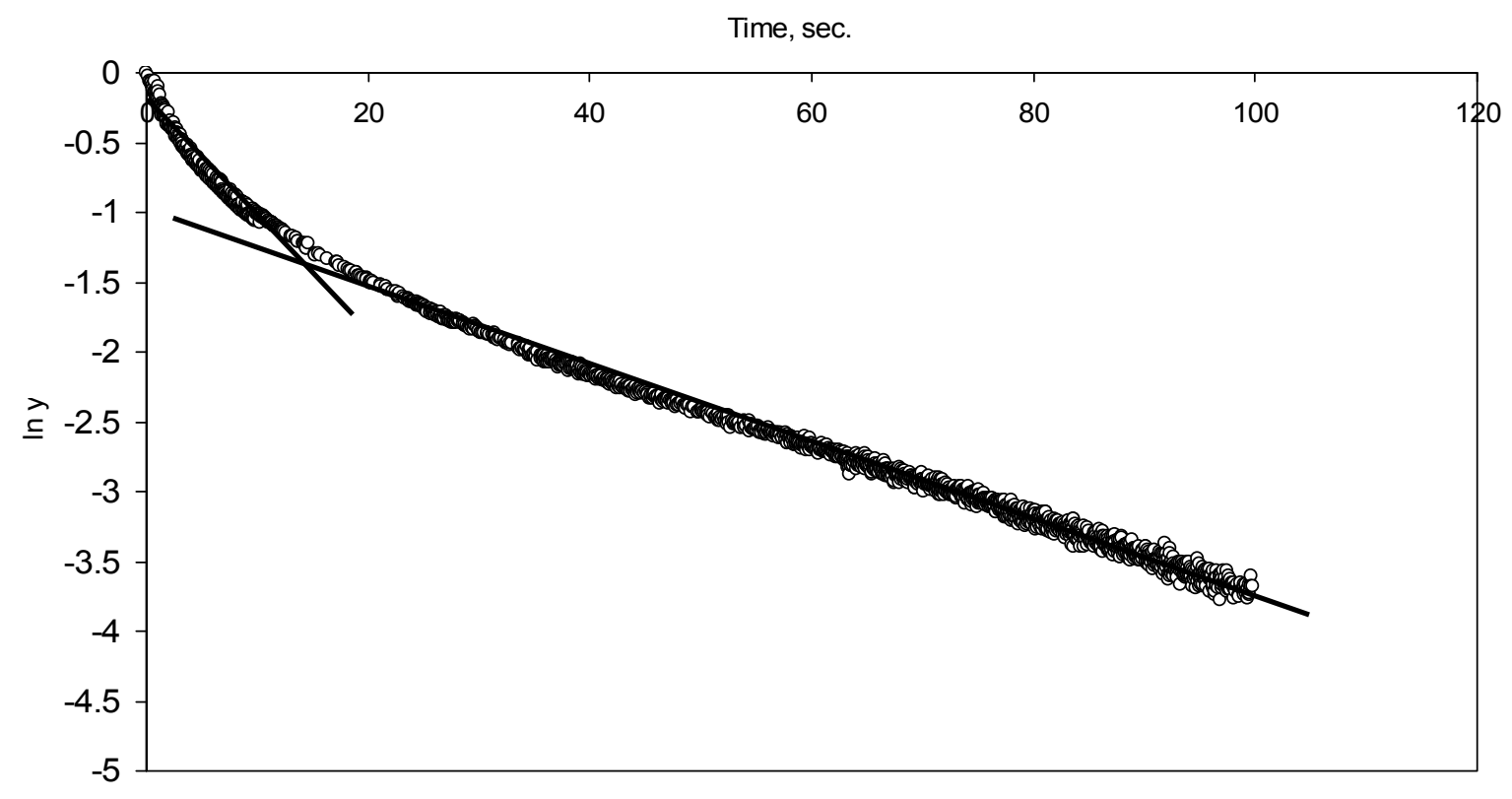

Fig. 2. Dependency of logarithm of $y$ on spreading time according to Eq. (4) for T6 solution at concentration $0.25 \mathrm{CWC}$. Fitted straight lines according to Eq. (4).

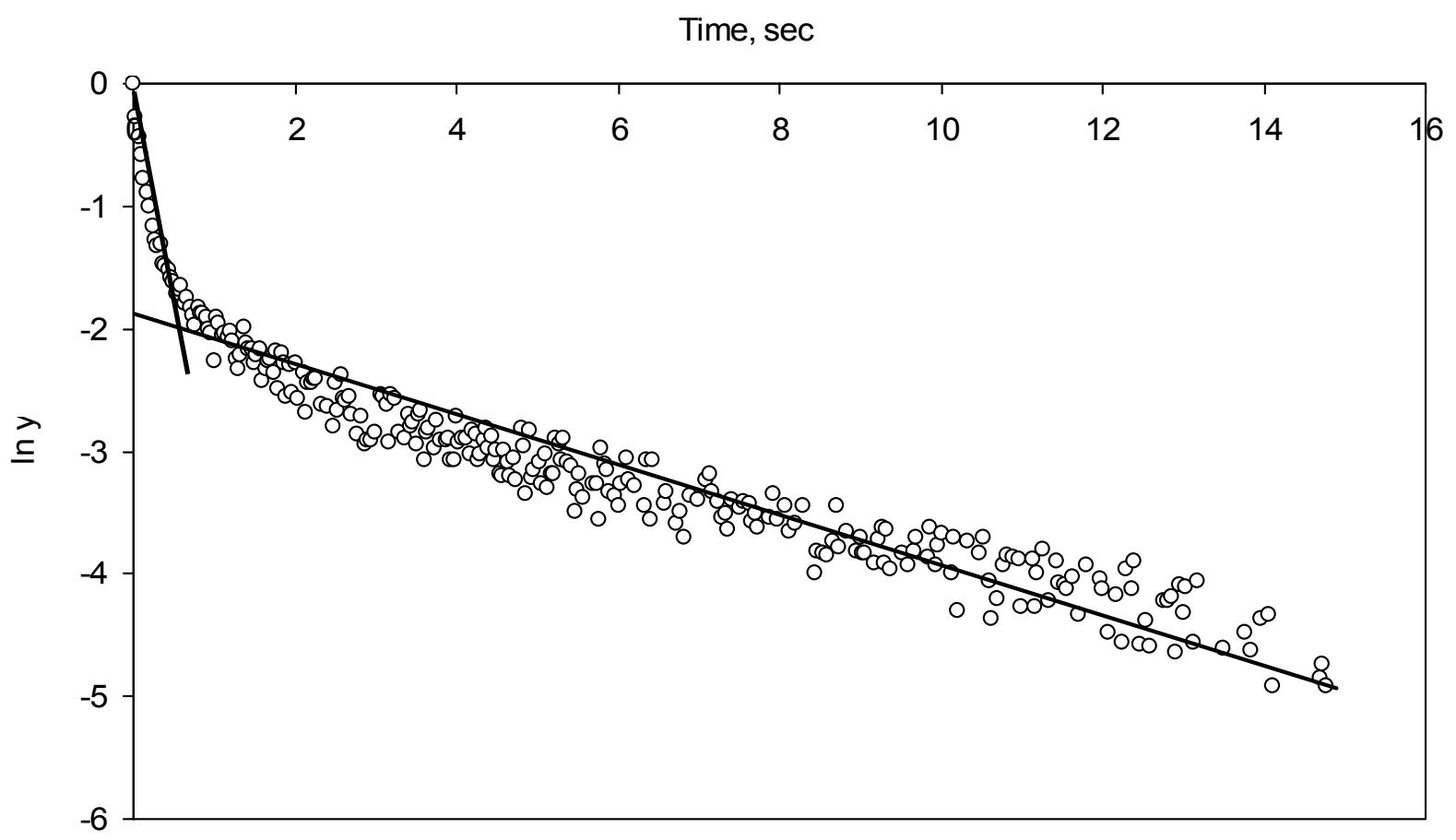

Fig.3. Dependency of logarithm of $y$ on spreading time according to Eq. (4) for T6 solution at concentration $5 \mathrm{CWC}$. Fitted straight lines according to Eq. (4). 

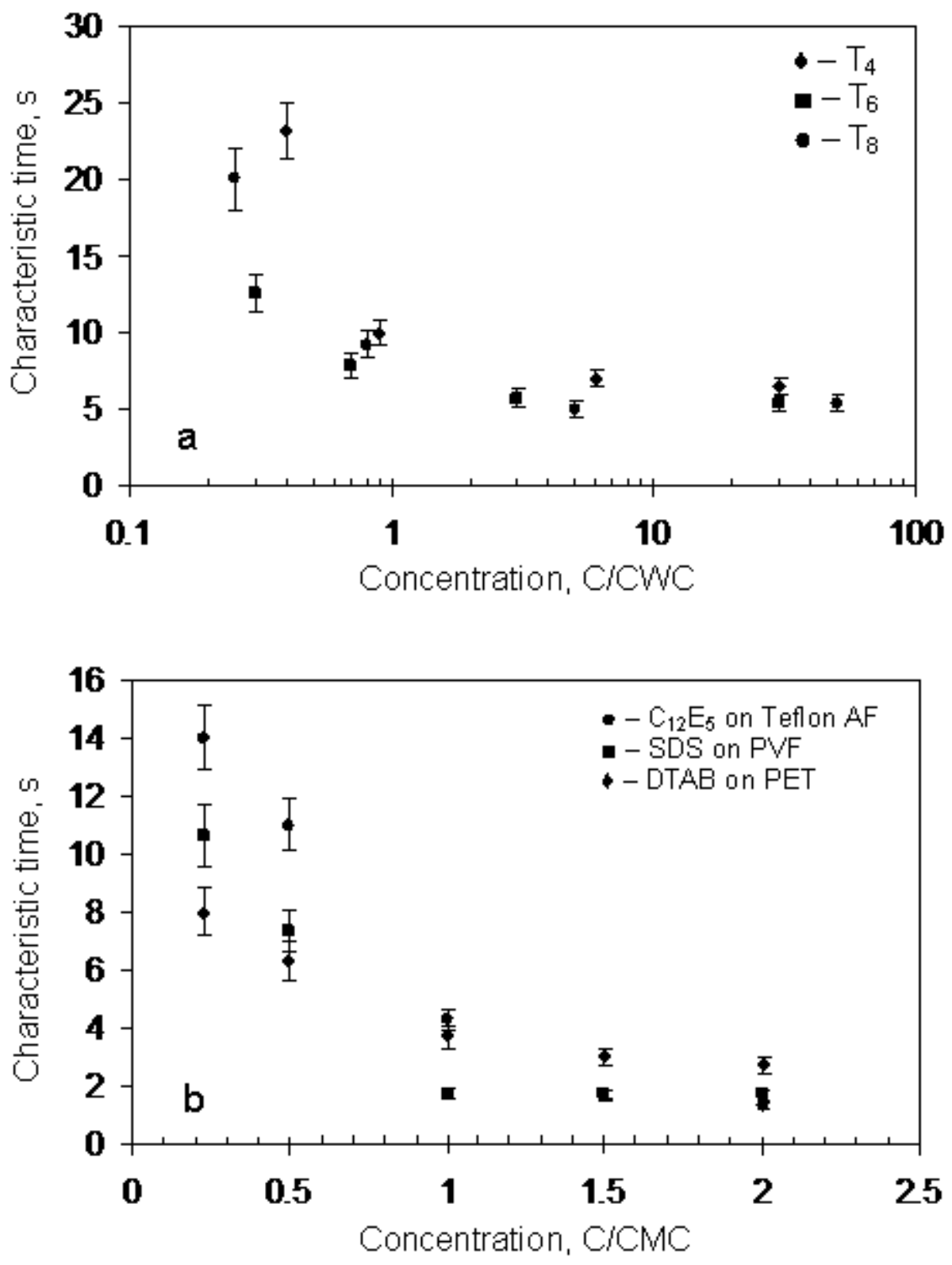

Figure 4. Dependencies of the time of surfactant transfer on a bare hydrophobic substrate in front of the three phase contact line on bulk concentration of surfactants: (a) trisiloxane surfactants $\left(T_{4}, T_{6}\right.$ and $\left.T_{8}\right)$ on PTFE AF coated silicone wafers; (b) (redrawn from Ref. ${ }^{14}$ ) conventional ionic (SDS, DTAB) and non-ionic $\left(\mathrm{C}_{12} \mathrm{E}_{5}\right)$ surfactants on different polymers. 


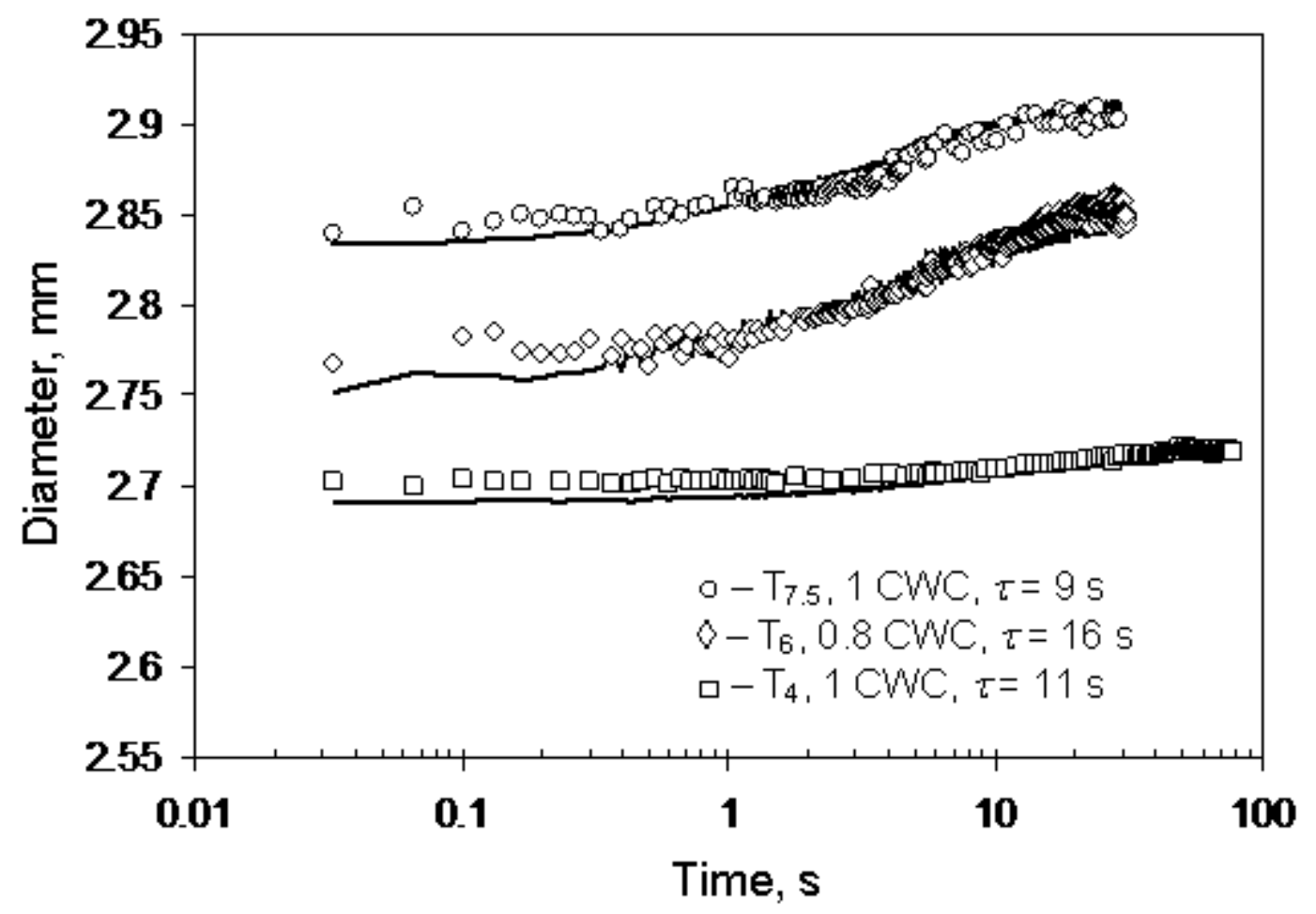

Figure 5. Time evolution of the droplet diameters for $T_{7.5}, T_{6}$, and $T_{4}$ solutions at concentrations close to the CWC (a second stage of spreading). Solid lines according to theoretical predictions (Refs. ${ }^{15,23}$ ) using the fitted values of the time of surfactant transfer. 


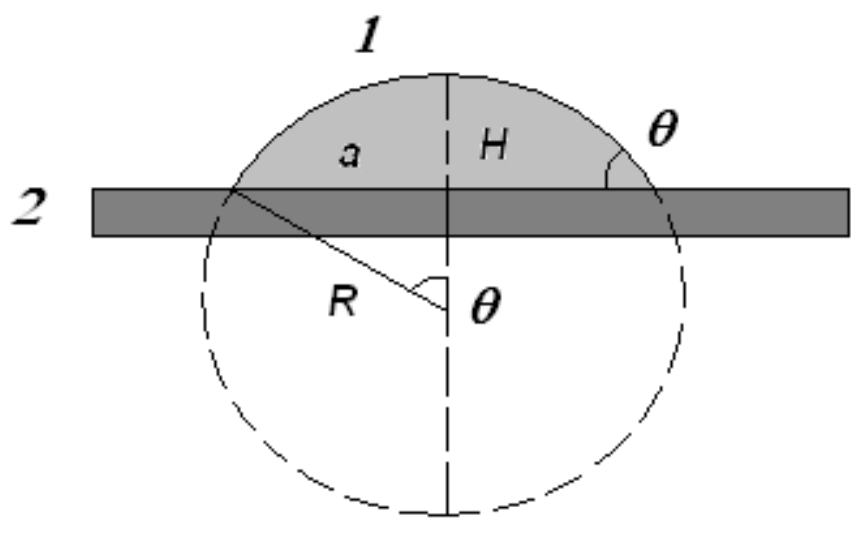

Figure 6. Liquid droplet 1 on a solid substrate 2. a- radius of the droplet base, $R$ radius of the curvature, $\boldsymbol{H}$-height, $\boldsymbol{\theta}$ - contact angle. 

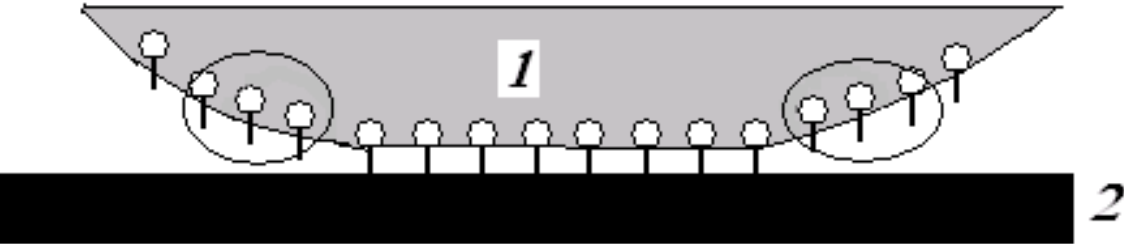

Figure 7a. A droplet of aqueous surfactant solution on a highly hydrophobic substrate. 1 - droplet, 2 - solid substrate. Hydrophobic tails of circled surfactant molecules attracted to the hydrophobic substrate and as results of thermal fluctuations some of those molecules can jump down on the bare hydrophobic substrate.

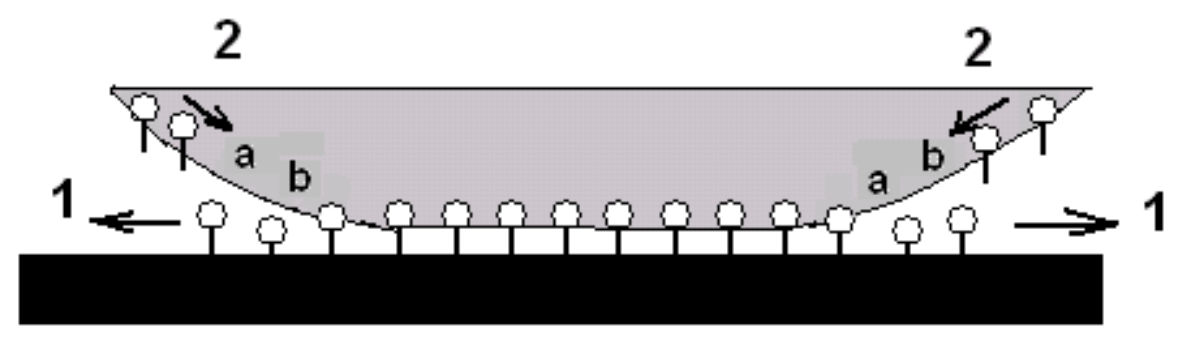

Figure $7 \mathrm{~b}$. Jump of a surfactant molecule from the liquid-air interface onto the solidair interface caused by thermal fluctuations. Three processes starts simultaneously but with different characteristic time scales: 1- surface diffusion along the solid surface, 2- Marangoni flow caused by the surface tension gradient, 3- attraction of the free part of the surface $(a b)$ and hydrophilic parts of the surfactant molecules underneath. 


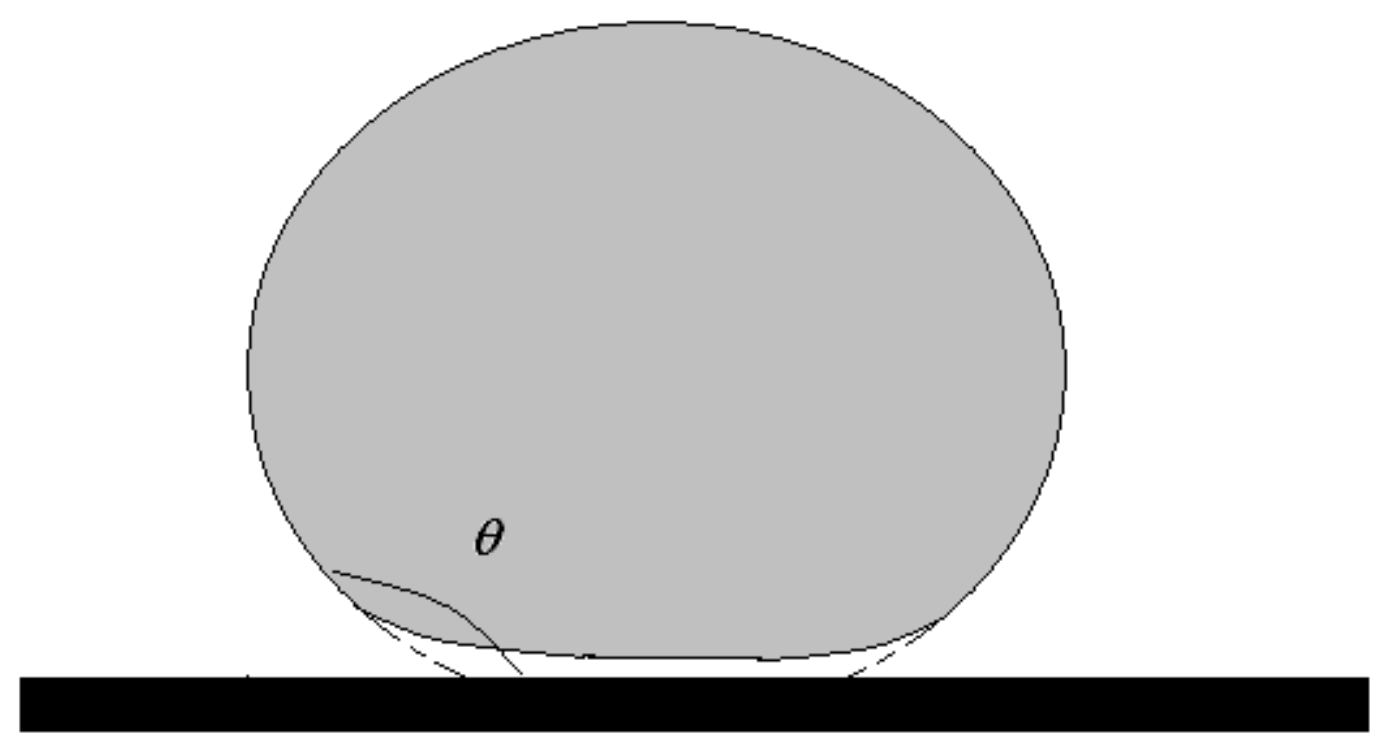

Figure 8a. Transition region from flat region in the centre to the droplet in the case of contact angle $\theta>90^{\circ}$.

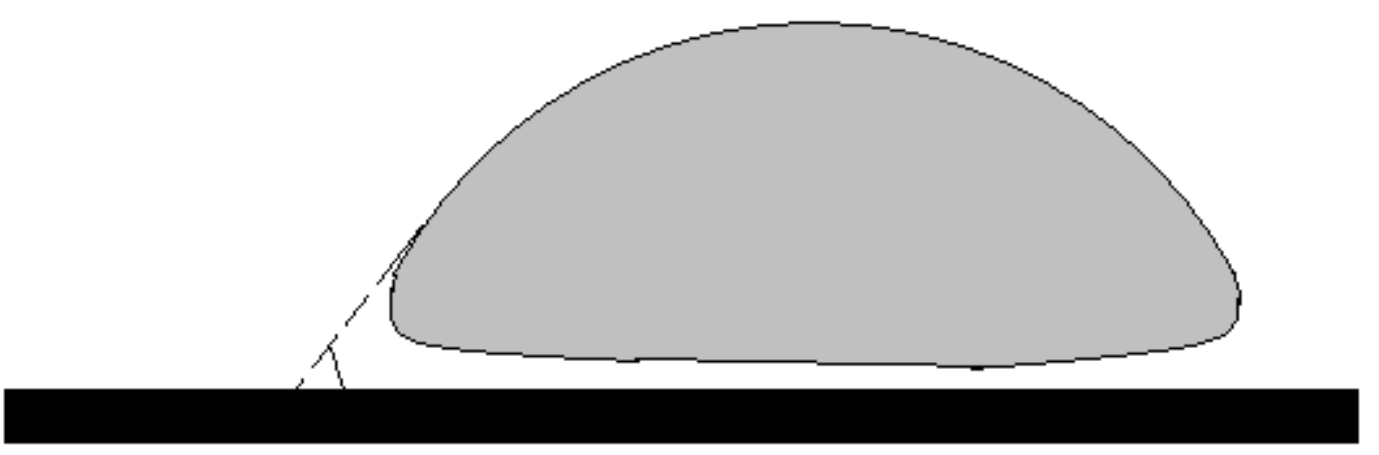

Figure $8 \mathrm{~b}$. Transition region from flat region in the centre to the droplet in the case of contact angle $\theta<90^{\circ}$. 


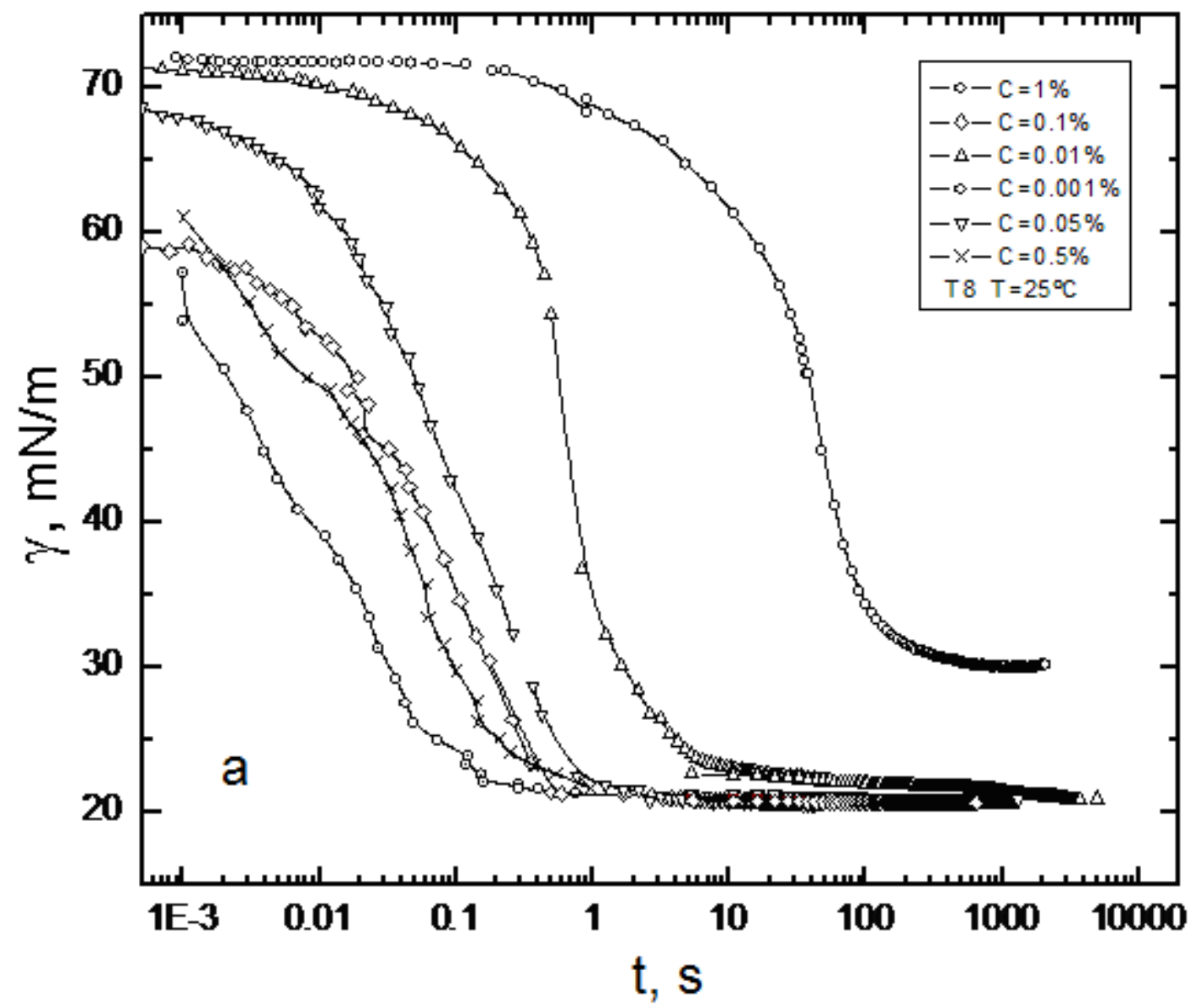

Figure 9. Dynamic surface tensions for T8 solutions at $298.15 \mathrm{~K}$ as a function of time. The symbols are experimental data, and the lines are eye-guides. 


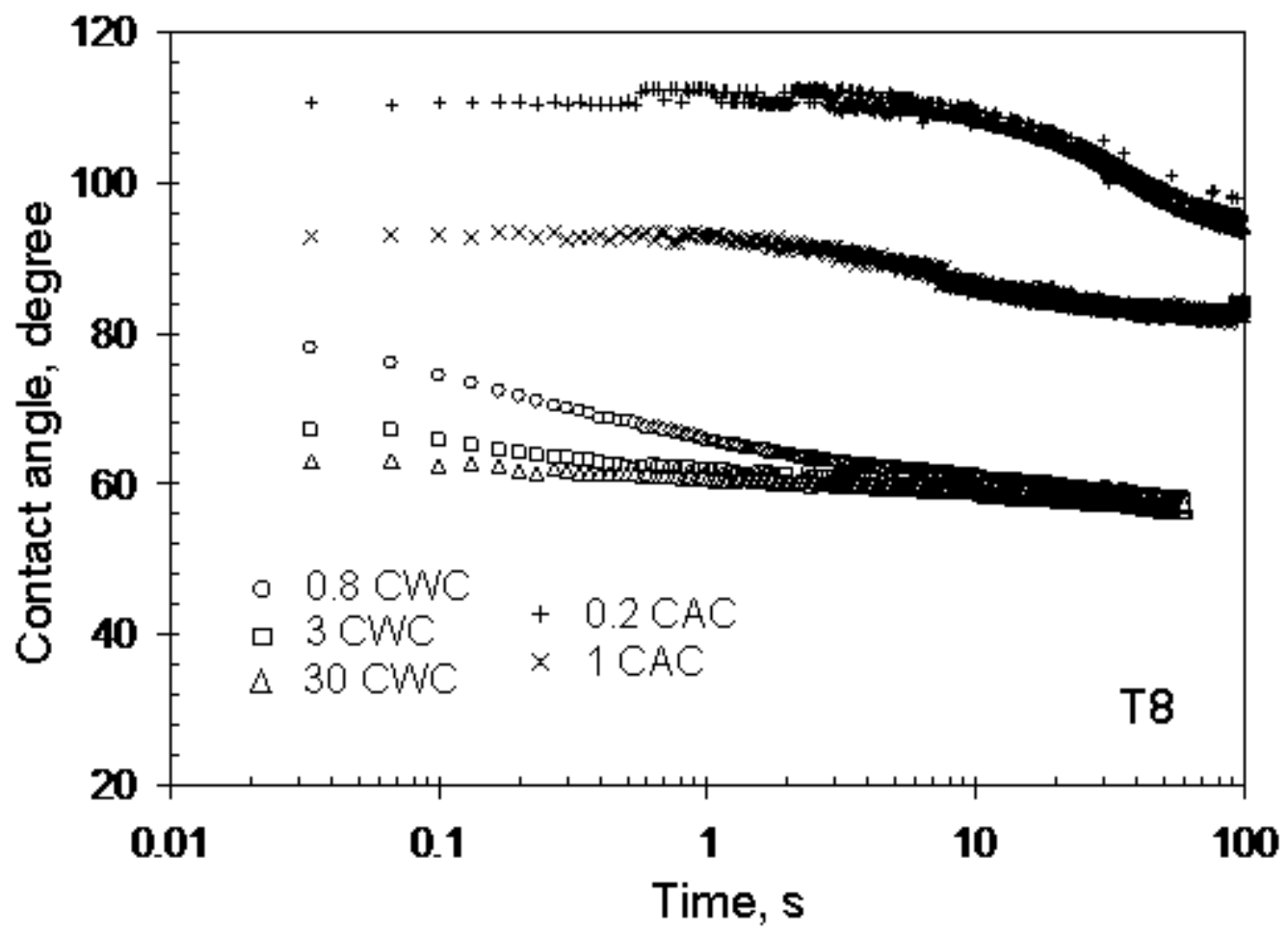

Figure 10. Dynamic contact angle for T8 solutions. 


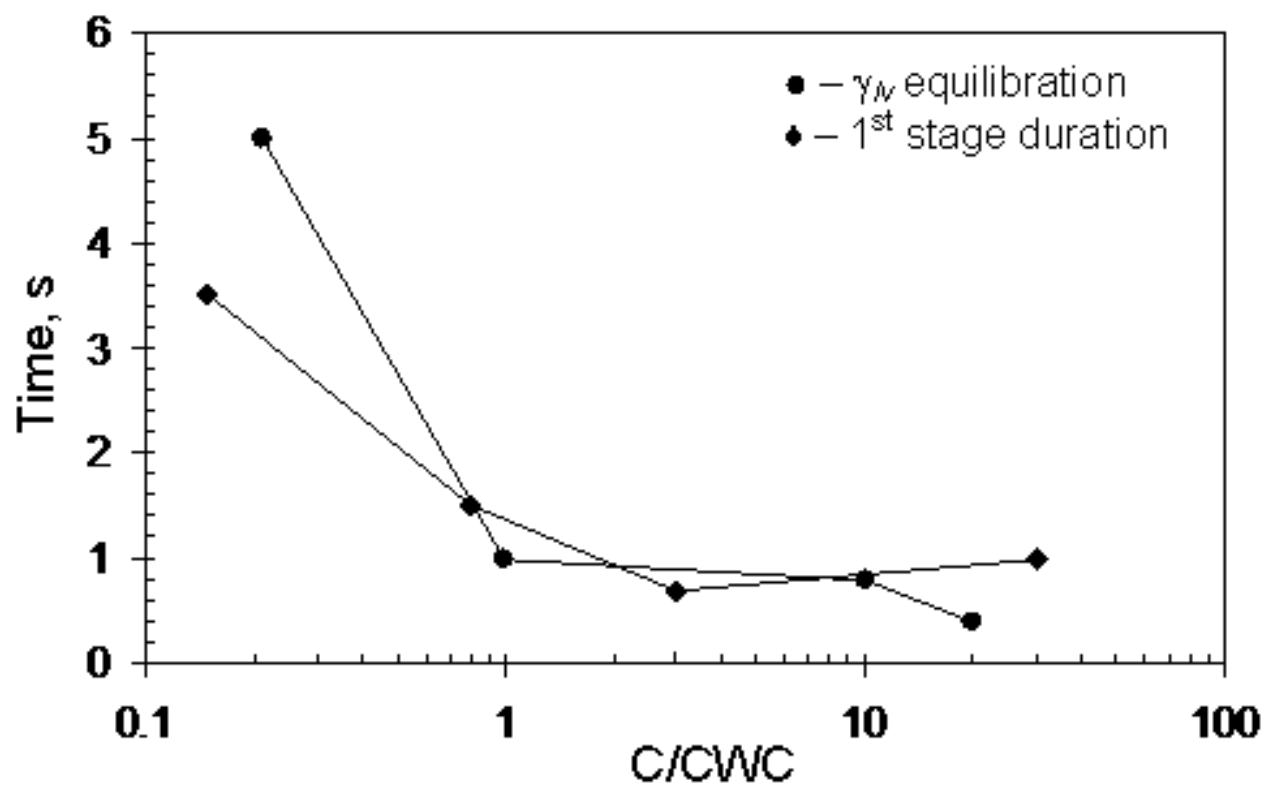

Figure 11. Dependency of the time of the surface tension equilibration for T8 $(\bullet)$, the duration of the fist stage of spreading droplets on PTFE AF coated silicon wafers from $\operatorname{ref}^{15}(\diamond)$. 